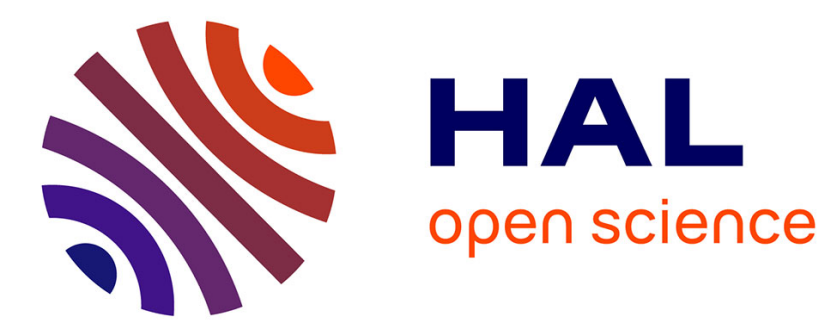

\title{
Photoluminescence from GaAs/AlGaAs quantum wires and quantum dots
}

\author{
K. Brunner, U. Bockelmann, G. Abstreiter, M. Walther, G. Böhm, G. \\ Tränkle, G. Weimann
}

\section{- To cite this version:}

K. Brunner, U. Bockelmann, G. Abstreiter, M. Walther, G. Böhm, et al.. Photoluminescence from GaAs/AlGaAs quantum wires and quantum dots. Journal de Physique IV Proceedings, 1993, 03 (C5), pp.C5-107-C5-114. 10.1051/jp4:1993519 . jpa-00251605

\section{HAL Id: jpa-00251605 https://hal.science/jpa-00251605}

Submitted on 1 Jan 1993

HAL is a multi-disciplinary open access archive for the deposit and dissemination of scientific research documents, whether they are published or not. The documents may come from teaching and research institutions in France or abroad, or from public or private research centers.
L'archive ouverte pluridisciplinaire HAL, est destinée au dépôt et à la diffusion de documents scientifiques de niveau recherche, publiés ou non, émanant des établissements d'enseignement et de recherche français ou étrangers, des laboratoires publics ou privés. 


\title{
Photoluminescence from GaAs/AlGaAs quantum wires and quantum dots
}

\author{
K. BRUNNER, U. BOCKELMANN, G. ABSTREITER, M. WALTHER, G. BÖHM, G. TRÄNKLE \\ and G. WEIMANN
}

Walter Schottky Institut, TU München, Am Coulombwall, 85748 Garching, Germany

\begin{abstract}
Quantum wires and dots have been fabricated by local interdiffusion of undoped GaAs/AlGaAs quantum well structures. Thermal interdiffusion is induced by a focused laser beam. In periodic arrays of quantum wires the photoluminescence (PL) splitts into several predominant lines which are separated by up to about $8 \mathrm{meV}$. These PL lines are attributed to the quantum number conserving optical transitions between 1dimensional electron and hole levels. Sample inhomogeneities cause broadening of the lines. The transition peaks shift in energy scanning a PL probe with a size of only one micron across the structures. Inhomogeneous line broadening is eliminated by investigation of a single quantum dot. A series of dots with different size show a systematic behaviour of PL blueshift and PL peak splitting. The main peaks in PL and PL excitation (PLE) spectra coincide well in energy and they are separated by up to about $10 \mathrm{meV}$. These peaks can be described by the allowed transitions between OD single particle levels within the nearly parabolic potentials caused by interdiffusion. Detailed calculations of excitonic states within the structures result in similar optical spectra. The observation of intense luminescence from excited dot levels indicates a slowing down of the energy relaxation of carriers. This is in good agreement with calculated LA phonon emission rates of carriers in OD systems. At low excitation density PL lines from a single quantum dot reveal widths of less than $0.5 \mathrm{meV}$.
\end{abstract}

Several techniques have been developped to fabricate semiconductor structures of reduced dimensionality. Quantum wire and dot structures revealing strong luminescence and absorption imply spatially direct optical transitions within a direct semiconductor, like the GaAs/AlGaAs material system. Various growth methods, strain patterning and defect-induced local interdiffusion of single quantum well samples have been shown to result in a lateral modulation of the bandgap localizing electrons as well as holes [1-9]. In our approach the bandgap is increased 
locally by interdiffusion of GaAs and AlGaAs layers, which is induced by the absorption of a focused laser beam. Usually the luminescence of low dimensional structures with transition peaks expected to be sharp is considerably broadened and smeared out by sample inhomogeneities. The influence of local potential fluctuations is analyzed by microscopic photoluminescence (PL) and PL excitation spectroscopy at liquid helium temperature with a spatial resolution of about $1.5 \mu \mathrm{m}$. This allows spectroscopy even of single quantum structures at low optical excitation density.

A schematic view of the experimental setup for the fabrication of lateral nanostructures is shown in Fig. 1. An $\mathrm{Ar}^{+}$-laser beam is stabilized in intensity to about $0.1 \%$ and is focused onto the sample. The diameter of the spherical spot of about $600 \mathrm{~nm}$ (1/e-value) is kept constant employing a sensitive autofocus system.

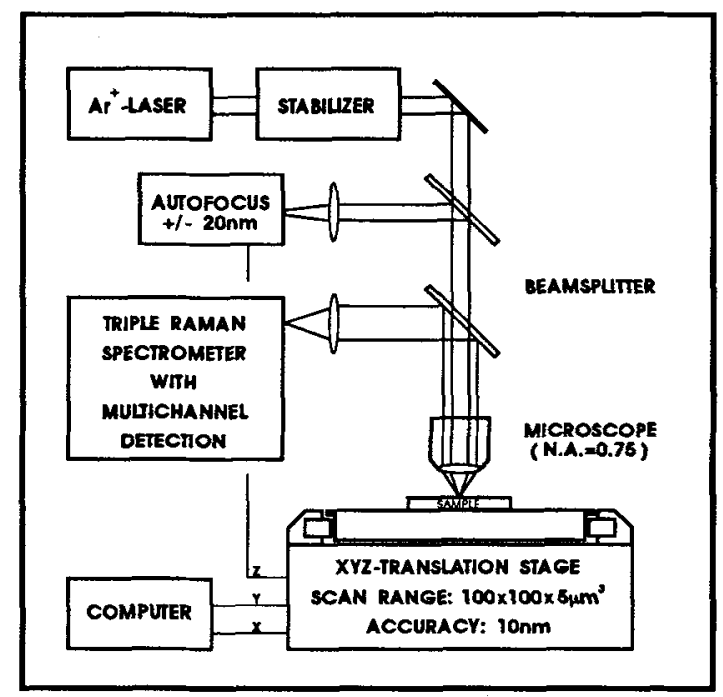

Fig. 1:

Schematic view of the experimental setup for fabrication of lateral nanostructures by laserinduced interdiffusion of a GaAs/AlGaAs quantum well sample.

Nearly arbitrary patterns can be drawn by internal interdiffusion induced by the strong absorption of the laser spot within a GaAs/AlGaAs quantum well sample which is scanned on a high precision xyz-translation stage. With a step size of $25 \mathrm{~nm}$ the positioning accuracy is about $10 \mathrm{~nm}$. The $\mathrm{Ga} / \mathrm{Al}$-interdiffusion process takes place at temperatures exceeding about $\mathrm{T}=900^{\circ} \mathrm{C}$ with a large thermal activation energy of about $\mathrm{E}_{\mathrm{a}}=5 \mathrm{eV}$. The temperature profile induced by the laser spot inside the samples has been calculated. At a laser power $P_{L}=6.6 \mathrm{~mW}$ we get a lateral interdiffusion profile, which is determined by the $\mathrm{Al} / \mathrm{Ga}$-diffusion coefficient [9-11]

$D_{A 1 / G a}(r)=3.0 \AA^{2} / s \cdot \exp \left\{-r^{2} /(60 n m)^{2}\right\}$ 
The extremely nonlinear increase of interdiffusion with local temperature results in an improvement of the lateral resolution by a factor of about 5 and in a strong dependance of interdiffusion on laser intensity. Due to the strong absorption the interdiffusion is limited to depths of about $500 \AA$ underneath the sample surface. Decomposition of the surface layers during laser processing is prevented by a plasma-deposited $\mathrm{Si}_{3} \mathrm{~N}_{4}$-film with a thickness of $1000 \AA$. The studies presented have been performed on a $30 \AA$-thick GaAs single quantum well embedded in $200 \AA$-thick $\mathrm{Al}_{0.35} \mathrm{Ga}_{0.65} \mathrm{As}$ barriers underneath a $100 \AA$-thick $\mathrm{GaAs}$ cap layer.

The microscope stage is optically coupled to a triple grating Raman spectrometer with a cooled Si-diode multichannel detection system. This offers a qualitative in-situ control of the current local sample properties by Raman spectroscopy of the phonon modes excited by the laser spot processing the sample. The Stokes/Antistokes intensity ratio, the frequency shifts and the broadening of the optical phonon lines monitor the local heating and interdiffusion of the quantum well layers averaged over the probed volume, which is given by the laser spot size and the laser penetration depth of about $1000 \AA$ [10]. The shift and broadening of the Raman lines caused by interdiffusion are more clearly observed in spectra measured at low excitation power after processing. Microscopic PL and PL excitation spectroscopy are performed at a temperature $\mathrm{T}=5 \mathrm{~K}$ using a cold-finger He-flow cryostat mounted on the translation stage. A HeNe laser or a tunable Ti-sapphire laser at a typical power of $1 \mu \mathrm{W}$ is used for excitation. PLE spectra are detected by a cooled photomultiplier tube. The probed lateral region is limited to about $1.5 \mu \mathrm{m}$ (1/e-diameter) by a confocal optical arrangement with a field aperture at an image point of the PL.

A series of quantum wire arrays with size $8 \mu \mathrm{m} \times 8 \mu \mathrm{m}$ has been fabricated by interdiffusing lineshaped barrier regions with different lateral separation a [10]. The luminescence from the $L=$ $30 \AA$ quantum well is nearly unaffected in arrays of large period length a $\geq 400 \mathrm{~nm}$. With decreasing period length the PL intensity maximum shifts to higher energy and some finestructure appears at the high energy side of the luminescence. At a period a $=200 \mathrm{~nm}$ five predominant $\mathrm{PL}$ lines are observed which are separated by about $8 \mathrm{meV}$. At this period a strong modulation of the $\mathrm{Al}$-content within the quantum well layer is reached with barely interdiffused regions in between the laser scanned lines. Such a wire structure is sketched ideally in Fig. 2. A typical PL spectrum measured with high spatial resolution is shown in the upper part of Fig. 3.

Some distinct PL lines are resolved probing only about 7 quantum wires at a length of about $1.5 \mu \mathrm{m}$. Increasing the probed area additional peaks appear from neighbouring lateral regions. The peaks merge to high energy shoulders which are considered to be characteristic of PL spectra from large areas of inhomogeneous quantum wire arrays. At smaller period a $\leq 150 \mathrm{~nm}$ interdiffusion between the lines written with the laser causes a strong PL blueshift and the local bandgap fluctuations increase. 


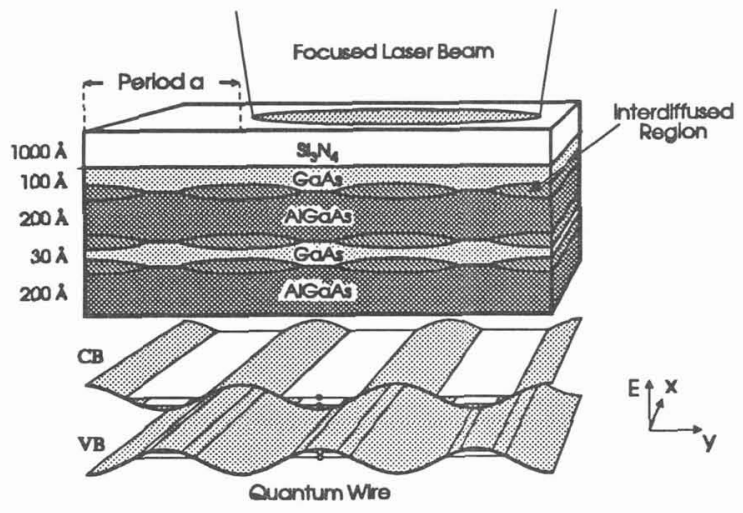

Fig. 2:

Schematic view of a quantum wire array fabricated by laserinduced interdiffusion of a $\mathrm{GaAs} / \mathrm{Al}_{0.35} \mathrm{Ga}_{0.65} \mathrm{As}$ quantum well structure $(L=30 \AA)$.

Fig. 3:

PL spectra of a wire array with period $a=200 \mathrm{~nm}$ measured with different probe size $2 \mathrm{r}_{\mathrm{a}}=1.5 \mu \mathrm{m}, 5 \mu \mathrm{m}$ and $8 \mu \mathrm{m}$.

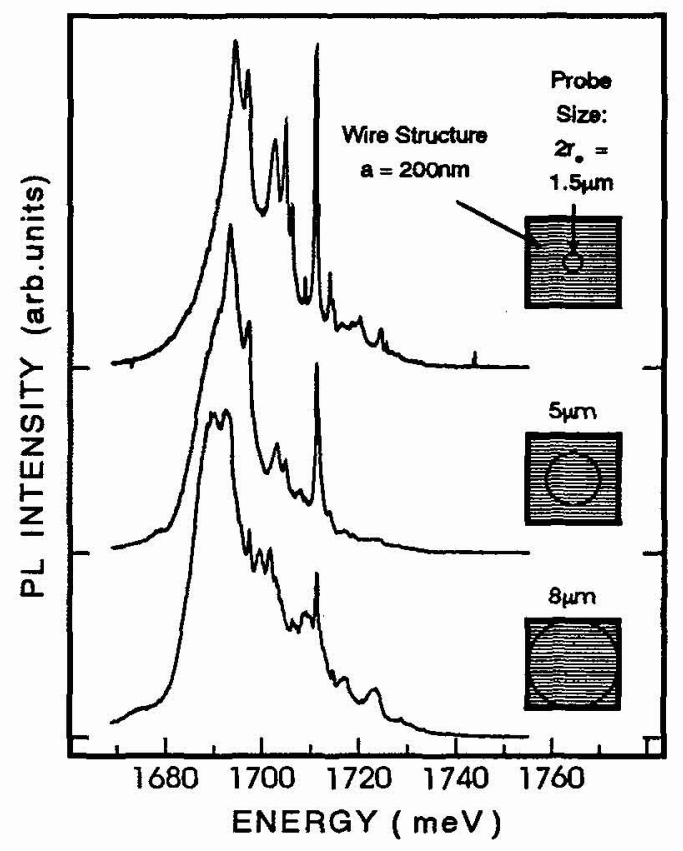

Using the lateral interdiffusion profile of eq. (1) we calculate the spatial modulation of the Al-content in the interdiffused quantum well assuming the interfaces to be smeared out as errorfunction-like profiles of Al-content. Separating the single-particle Schrödinger equation with $\psi_{\mathrm{e}, \mathrm{h}}$ $=\psi_{\mathrm{e} 0 \text {,hho }}(\mathrm{z}) \cdot \psi_{\mathrm{r}}(\mathrm{y}) \cdot \exp \left(\mathrm{ik}_{\mathrm{x}} \mathrm{x}\right)$ we obtain the 1-dimensional subbands near the band edges. At a period $\mathrm{a}=200 \mathrm{~nm}$ the groundstate is slightly shifted to higher energy and the subbands show a nearly constant energy separation of about $4.2 \mathrm{meV}$ and $3.2 \mathrm{meV}$ for electrons and holes, respectively. Similar results can be derived from model calculations for sinusoidal potentials including the mixing of heavy and light hole states [12]. The observed main peak splitting agrees reasonably well with the transition energies of the allowed radiative electron-hole recombination between 1D-subbands of equal quantum numbers. The luminescence properties of these quantum wire structures have been calculated by Glutsch and Bechstedt in a more detailed model including the excitonic binding of electron and hole [13,14]. The calculated spectra reveal nearly equidistant excitonic transition peaks at the high energy side whose energy separation increases with decreasing period a. At a period $a=200 \mathrm{~nm}$ distinct peaks are separated by roughly $8 \mathrm{meV}$. They may be attributed to the lateral center-of-mass localization of excitons with 1s-like envelope wavefunctions for the internal motion. The transition probability of the excited states is about $20 \%$ compared to the groundstate. The finestructure and additional peaks are probably due to 
local inhomogeneities which are more clearly resolved by the PL experiments at high spatial resolution.

The inhomogeneous broadening of luminescence lines can be eliminated by spectroscopy of a single quantum dot. Local fluctuations of the confining potential should only result in a lifting of level degeneracies and in a finestructure of the spectra with transition peaks of intrinsically homogeneous linewidths. A series of single quantum dots of geometrical size from $w=$ $250 \mathrm{~nm}$ to $2000 \mathrm{~nm}$ has been fabricated using a focused laser beam of constant intensity [15]. A schematic cross section of a dot structure and the intended lateral band edge modulation are shown in the inset of Fig.4.

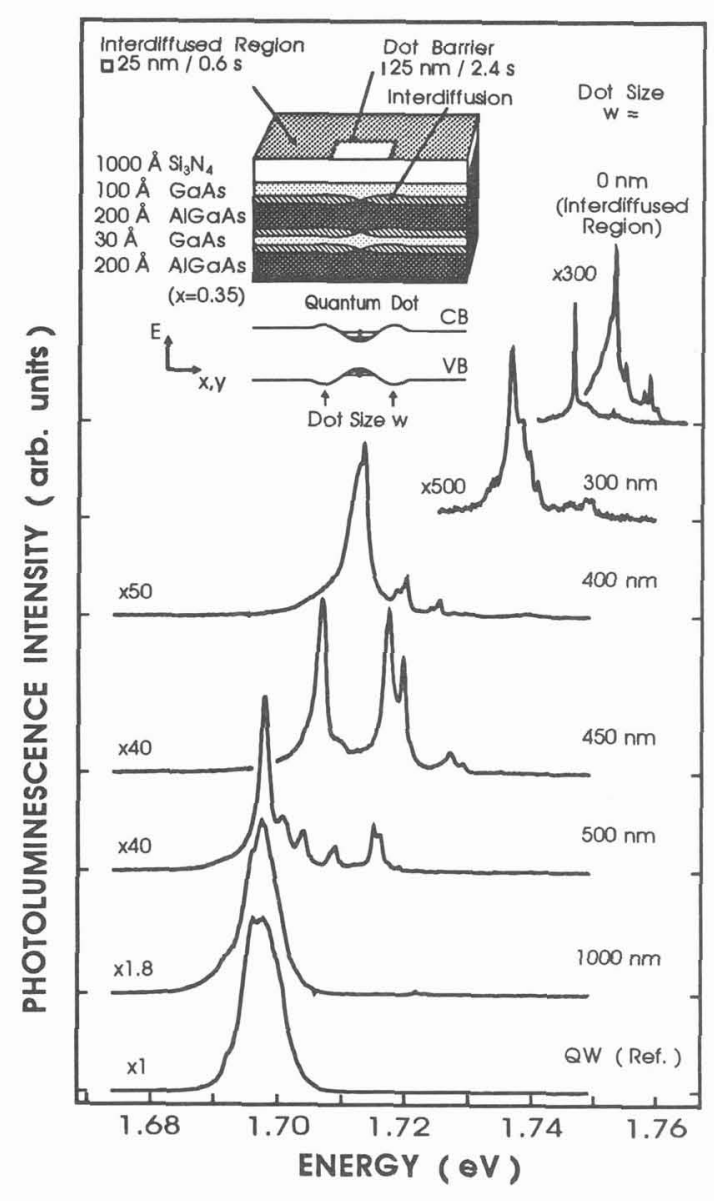

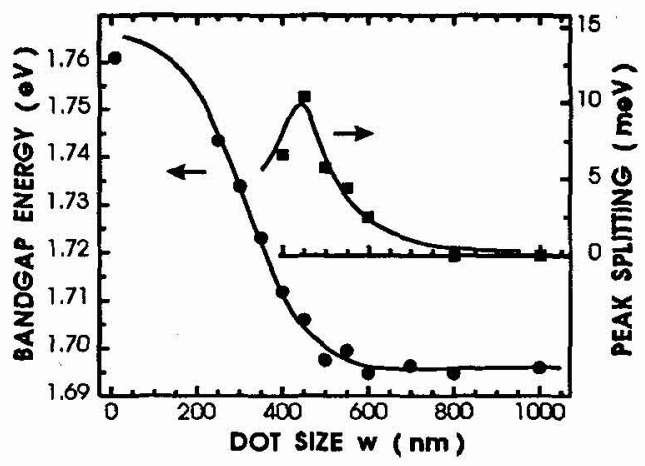

Fig. 4:

Microscopic PL spectra of single quantum dots of various size $w$. The exciting laser spot was $E_{\mathrm{Exc} .}=1.96 \mathrm{eV}, \mathrm{P}_{\mathrm{Bxc} .}=1 \mu \mathrm{W}$ and $2 \mathrm{r}_{\mathrm{e}}=1.5 \mu \mathrm{m}$. A schematic view of a dot structure fabricated by laser-induced interdiffusion of a $\mathrm{GaAs} / \mathrm{AlGaAs}$ quantum well and the intended band edge modulation are shown in the inset. From ref. [15].

Fig. 5:

Minimum energy of the PL peaks and energy separation of the predominant peaks in dependance of the geometrical dot size $w$. The solid lines are just a guide to the eye. From ref. [15].

The optically active dot is defined by a square-shaped lateral barrier of size $w \mathrm{x} w$ and it is surrounded by a nominally homogeneously interdiffused area which is $6 \mu \mathrm{m} \times 6 \mu \mathrm{m}$ in size. Based on Raman and PL spectra from test structures the power of the processing laser spot has been 
chosen so high that only a very weak, strongly blueshifted luminescence can be observed from this interdiffused area. The special design of the single dot structure allows a clear identification of the locally luminescing dot at the center of a structure. Some typical microscopic PL spectra from dots of different size are shown in Fig 4. The unprocessed $30 \AA$-thick quantum well emits an inhomogeneously broadened line with a spectral width of about $7 \mathrm{meV}$. The small peaks and shoulders near the luminescence maximum change with the lateral position of the PL microprobe and they are attributed to excitons which are localized at minima of the effective bandgap within the thin GaAs quantum well. From large dots $w \geq 800 \mathrm{~nm}$ similar PL spectra are observed. With decreasing dot size a more and more pronounced peak structure appears at the high energy side. At a dot size $w=450 \mathrm{~nm}$ the luminescence is blueshifted by about $12 \mathrm{meV}$ and intense luminescence peaks are observed at higher energy. The predominant peaks are nearly equidistant and they are separated by about $10 \mathrm{meV}$. With further decreasing dot size the PL shifts to higher energies concomitant with a decrease in the peak splitting. Within the interdiffused areas surrounding the dots PL can be observed only at some locations, which is weak and blueshifted by up to about $70 \mathrm{meV}$ ( $w=0 \mathrm{~nm}$ in Fig. 4). This energy gives roughly a lower estimate of the bandgap energy of the topmost totally interdiffused layers. The energy of the fundamental PL peak and the energy separation of the PL main peaks in dependance on dot size are summarized in Fig. 5. The PL shifts monotonically to higher energy when decreasing the dot size below 500nm. At the onset of the blueshift the maximum peak splitting is observed. These PL features can be qualitatively understood by the expected effective lateral modulation of the band edges, which is shown in Fig. 6 for $w=600 \mathrm{~nm}, 450 \mathrm{~nm}$, and 300nm.

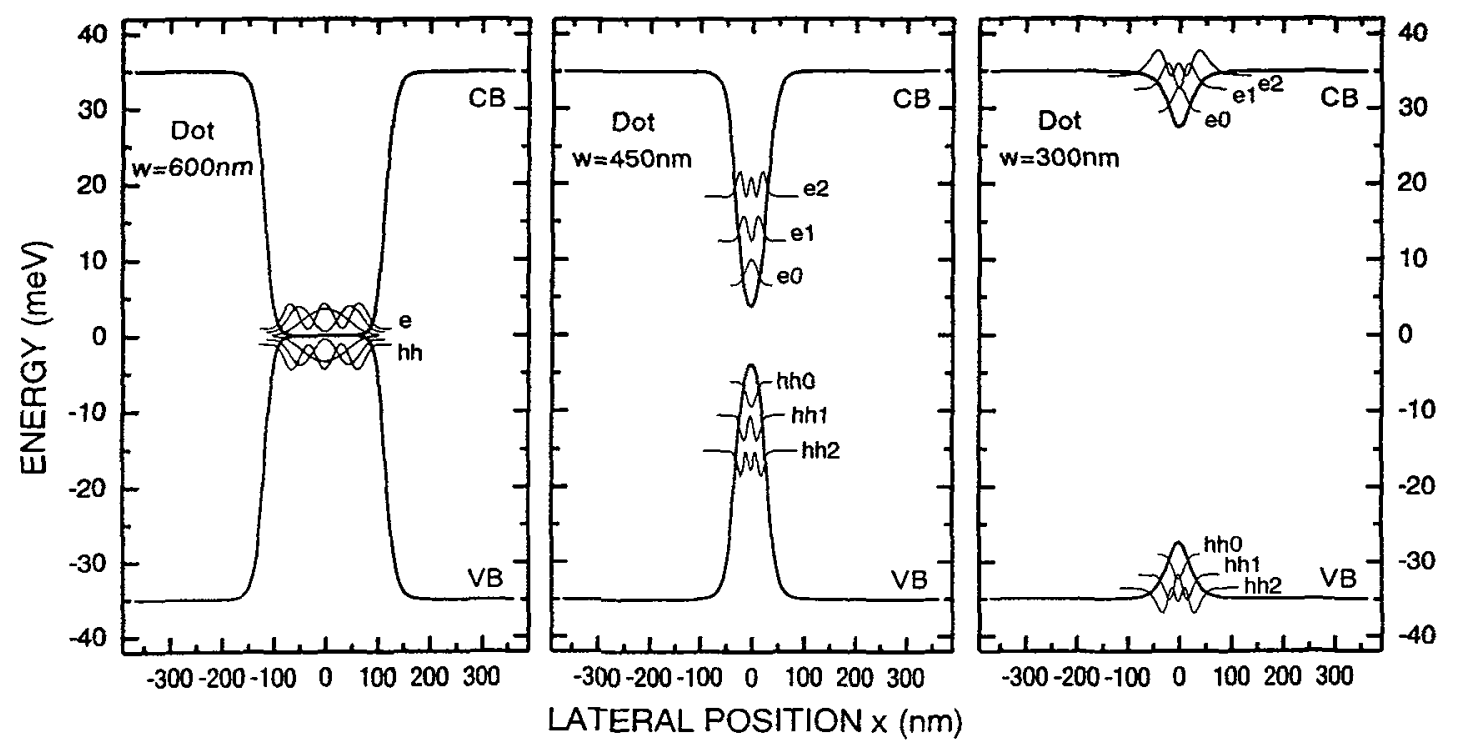

Fig. 6:

Lateral modulation of the effective band edge energies, calculated for single quantum dots of geometrical size $w=600 \mathrm{~nm}, 450 \mathrm{~nm}$, and $300 \mathrm{~nm}$. Energy $E=0$ corresponds to the fundamental electron and heavy hole subbands within the as-grown quantum well. The three lowest levels of electrons and heavy holes are shown. 
With increasing interdiffusion of the $\mathrm{GaAs} / \mathrm{Al}_{0.35} \mathrm{Ga}_{0.65} \mathrm{As}$ quantum well with thickness $\mathrm{L}=$ $30 \AA$ the energies of the lowest electron and heavy hole quantum well subbands shift to larger values. The calculated shifts differ only within about $20 \%$. Thus the lateral modulation of the effective conduction and valence band edge are assumed to be equal with a maximum height of $35 \mathrm{meV}$ corresponding to the maximum blueshift observed. At large dot size $w=600 \mathrm{~nm}$ the lateral quantization is neglegibly small. At a dot size of $w=450 \mathrm{~nm}$ the effective lateral potential is nearly parabolic and of rotational symmetry. So the envelope wavefunctions shown in Fig. 6 in $x$ direction also apply in y-direction and the single particle levels $e_{r}$ and $h h n_{r}\left(n_{r}=0,1,2 \ldots\right)$ are of degeneracy $2\left(\mathrm{n}_{\mathrm{r}}+1\right)$ including spin. The lateral wavefunctions are localized within about $30 \mathrm{~nm}$ at the dot center and the quantization energies are about $n_{r} \cdot \hbar \omega_{e, h}$ with energies $\hbar \omega_{e}=6 \mathrm{meV}$ and $\hbar \omega_{h}$ $=4 \mathrm{meV}$. The observed PL peak splitting of $10 \mathrm{meV}$ agrees well with the expected energy separation of transitions between totally localized electron and hole levels of equal quantum numbers. In this dot the PL blueshift is mainly caused by the lateral quantization. Contrary to this the shift of the PL in a small dot $w=300 \mathrm{~nm}$ is dominated by interdiffusion at the dot center. The energy modulation height and the localization energies are decreased. Calculating the levels in a quantum dot with axialsymmetrical parabolic potential including the electron-hole Coulomb interaction results in strong mixing of single particle states forming excitonic levels [16]. Modelling the dot $w=450 \mathrm{~nm}$ results in optical transition peaks with a nearly constant energy separation which is comparable to the noninteracting case. They are attributed to the localized s-excitons with vanishing angular momentum $(j=0)$. Mixing of radiative excitons with $j=0$ and excitons of momentum $j \neq 0$ by a nonuniformly shaped potential can result in the spectral finestructure of excited states which is observed for example at energies around $E=1720 \mathrm{meV}$ in the PL of the dot $w=450 \mathrm{~nm}$ in Fig. 4 .

The observation of the strongest PL from excited dot levels at the maximum peak splitting of $10 \mathrm{meV}$ implies a nonthermal level occupation within the dot $w=450 \mathrm{~nm}$. This is attributed to the quenching of the LA phonon emission rate in quantum dots with quantization energies exceeding a threshold value which is given by $E_{t}=\hbar c_{s} \cdot 2 \pi / L=5.1 \mathrm{meV}$, for $\mathrm{L}=30 \AA$ and the GaAs sound velocity $c_{s}=3700 \mathrm{~m} / \mathrm{s}[15,17,18]$. At a very low optical excitation corresponding to less than one electron-hole-pair on time average within the dot $w=450 \mathrm{~nm}$ the spectral width of individual PL peaks is below about $0.5 \mathrm{meV}$. With increasing excitation the peaks broaden. This can not be explained by phonon scattering and by crystal heating with the focused laser beam. Carrier-carrier scattering and energy shifts of the luminescence transitions due to a temporally fluctuating occupation of higher dot levels might explain the finite linewidths observed.

The main PL peaks are also observed in PL excitation (PLE) spectroscopy at the same energy positions within the experimental accuracy. PLE intensity peaks with energies corresponding to the PL shoulders in between the predominant PL peaks, which are attributed to the two lowest electron levels of the dot $w=450 \mathrm{~nm}$, are observed. They might be attributed to electronhole recombination with a change of the lateral quantum numbers (hh1,hh2 $\ldots \rightarrow \mathrm{e} 0$ ). This small 
energy splitting, however, is not reproduced within the excitonic model. The PLE spectra are strongly affected by the slow electron relaxation and they do not directly represent the absorption spectra in OD systems.

This work has been supported in part by the Bundesministerium für Forschung und Technologie (DFE Verbundprogramm), the Deutsche Forschungsgemeinschaft and a Procope contract.

[1] D. Gershoni, J. S. Weiner, S. N. G. Chu, G. A. Baraff, J. M. Vandenberg, L. N. Pfeiffer, K. West, R. A. Logan und T. Tanbun-Ek, Phys. Rev. Lett. 65, 1631 (1990).

[2] J. A. Lebens, C. S. Tsai, K. J. Vahala und T. F. Kuech, Appl. Phys. Lett. 56, 2642 (1990).

[3] W. Beinstingl, Y. J. Li, H. Weman, J. Merz und P. M. Petroff, J. Vac. Sci. Technol. B9, 3479 (1991).

[4] J. S. Weiner, D. Danan, A. Pinczuk, J. Valladares, L. N. Pfeiffer und K. West, Phys. Rev. Lett. 63, 1641 (1989).

[5] E. Kapon, D. M. Hwang und R. Bhat, Phys. Rev. Lett. 63, 430 (1989).

[6] K. Kash, B. P. Van der Gaag, D. D. Mahoney, A. S. Gozdz, L. T. Florez, J. P. Harbison und M. D. Sturge, Phys. Rev. Lett. 67, 1326 (1991).

[7] G. Mayer, B. E. Maille, R. Germann, A. Forchel, P. Grambow und H. P. Meier, Appl. Phys. Lett. 56, 2016 (1990).

[8] Y. J. Li, M. Tsuchiya und P. M. Petroff, Appl. Phys. Lett. 57, 472 (1990).

[9] H. Leier, A. Forchel, B. E. Maile und G. Weimann, Microelectr. Engineer. 9, 361 (1989).

[10] K. Brunner, G. Abstreiter, M. Walther, G. Böhm, und G. Tränkle, Proc. of the 5th Conference on Modulated Semiconductor Structures, (Nara 1991), Surf. Sci. 267, 218 (1992).

[11] E. Liarokapis und Y. S. Raptis, J. Appl. Phys. 57, 5123 (1985).

[12] U. Bockelmann und G. Bastard, Phys. Rev. B45, 1688 (1992).

[13] S. Glutsch und F. Bechstedt, Phys. Rev. B47, 6385 (1993).

[14] S. Glutsch und F. Bechstedt, Phys. Rev. B47, 4315 (1993).

[15] K. Brunner, U. Bockelmann, G. Abstreiter, M. Walther, G. Böhm, G. Tränkle und G. Weimann, Phys. Rev. Lett. 69, 3216 (1992).

[16] U. Bockelmann, (to be published).

[17] U. Bockelmann and G. Bastard, Phys. Rev. B42, 8947 (1990).

[18] H. Benisty, C. M. Sottomayor-Torres, and C. Weisbuch, Phys. Rev. B44, 10945 (1991). 